\title{
Fuzzy Logic Based Equivalent Consumption Optimization of a Hybrid Electric Propulsion System for Unmanned Aerial Vehicles
}

\author{
Ye Xie $^{\mathrm{a}, *}$, Al Savvaris ${ }^{\mathrm{a}}$, Antonios Tsourdos $^{\mathrm{a}}$ \\ ${ }^{a}$ School of Aerospace, Transport and Manufacturing, Cranfield University, Bedford, MK43 OAL, United \\ Kingdom
}

\begin{abstract}
This paper presents an energy management strategy for a hybrid electric propulsion system designed for unmanned aerial vehicles. The proposed method combines the Equivalent Consumption Minimization Strategy (ECMS) and fuzzy logic control, thereby being named Fuzzy based ECMS (F-ECMS). F-ECMS can solve the issue that the conventional ECMS cannot sustain the battery state-of-charge for on-line applications. Furthermore, F-ECMS considers the aircraft safety and guarantees the aircraft landing using the remaining electrical energy if the engine fails. The main contribution of the paper is to solve the deficiencies of ECMS and take into consideration the aircraft safely landing, by implementing F-ECMS. Compared with the combustion propulsion system, the hybrid propulsion system with F-ECMS at least reduces $11 \%$ fuel consumption for designed flight missions. The advantages of F-ECMS are further investigated by comparison with the conventional ECMS, dynamic programming and adaptive ECMS. In contrast with ECMS and dynamic programming, F-ECMS can accomplish a balance between sustaining the battery state-of-charge and electric energy consumption. F-ECMS is also superior to the adaptive ECMS because there are less fuel consumption and lower computational cost.
\end{abstract}

Keywords: UAV; Hybrid Electric Propulsion System; Equivalent Consumption; Optimization; Fuzzy Logic Control; Hamiltonian function

\section{Introduction}

Over the last decades, the rapid growth in consumption of fossil fuel, and resulting polluting emissions has generated a need for energy conservation. NASA N+3 concepts aim to reduce aircraft fuel burn by $60 \%$ by 2025 [1]. To meet this challenge, the aeronautic industries show great interest in electric propulsion systems. However, electric aircraft cannot achieve the same flight endurance as their conventional fuel-powered counterparts, due to lower-energy-density storage of sources such as batteries.

The Hybrid Electric Propulsion System (HEPS) is a new-generation concept, which combines an electric powertrain with an Internal Combustion Engine (ICE) to provide the propulsive power [2]. The result is decreased fuel burn compared with ICE-powered aircraft, and an increased flight range compared with electric aircraft. Thus, there is significant research interest in this technology and its applications for aircraft.

Initially, academics focused on the Unmanned Aerial Vehicle (UAV) to validate and demonstrate the feasibility of hybrid electric technology. The Air Force Institute of Technology began its investigations with

\footnotetext{
* Corresponding author

Email address: ye.xie@cranfield.ac.uk
} 
conceptual design and configuration comparisons of the hybrid system, see Harmon [3] and Hiserote [4]. It was concluded that the clutch-start parallel configuration is the most practically realizable. The research team from Queensland University of Technology also developed parallel HEPS by combing a 10 cc combustion engine and a $600 \mathrm{~W}$ brushless motor. Simulation and ground tests demonstrated that the aircraft fuel usage decreased by $6 \%$ with only a 5\% weight penalty compared to the non-hybrid system [5]. (Quasi-) static models of each component were developed for the preliminary design and sizing of HEPS, by Schoemann in the Technical University of Munich [6]. Panagiotou et al. studied the conceptual design of a hybrid UAV that could be electric-powered by both batteries and solar cells. The designed hybrid solar UAV has 50\% increased endurance during summertime [7]. Friedrich and Robertson, from the University of Cambridge, researched design and sizing methods for hybridelectric propulsion of a $20 \mathrm{~kg}$ UAV where the combustion engine was sized based on the engine ideal operating line [8]. Riboldi applied an optimal approach to design the small hybrid electric aircraft and investigated the sensitivity of the optimization results [9].

It is concluded from these studies that HEPS can provide better fuel economy and on-board electrical regeneration with minimal impact on the UAV's performance. However, this flexibility comes at the cost of increased complexity, i.e., the need to implement energy management.

Energy management of the hybrid propulsion system, also referred to as supervisory control, has the objective of splitting power requests between the combustion and electric powertrain. An example of a promising supervisory control is that proposed by Paganelli [10], the Equivalent Consumption Minimization Strategy (ECMS). The concept of equivalent consumption is a sum of actual fuel consumption of the ICE and the equivalent electrical energy consumption. Using the equivalent consumption, the ECMS can transform a global optimization problem into an instantaneous optimization problem. The new instantaneous optimization problem does not rely on a-priori global knowledge, has much less computational complexity and can be implemented for on-line energy management.

Conventionally, the ECMS estimates the equivalent consumption on-line using a pre-determined equivalence factor. The key issue with this approach is that it cannot sustain the battery State-of-Charge (SoC) due to the equivalence factor being evaluated off-line [11]. The Adaptive-ECMS (A-ECMS) [12,13], has been adopted as a charge-sustaining strategy to maintain the battery SoC at a specific value (such as $30 \%$ ), which is typical of hybrid ground vehicles [14]. However, the charge-sustaining strategy is not ideal for aerial vehicle applications [15]. If the SoC is sustained at a very low level, the residual battery capacity cannot guarantee a safe landing if there is a failure of the engine; on the other hand, maintaining a high-level SoC means full use cannot be made of the electrical energy in the battery. Therefore, for aircraft, it is better that the $\mathrm{SoC}$ will be held within a range instead of at a specific value. In sum, the conventional ECMS and its reformed approach (e.g. A-ECMS) have two issues when being applied to the aircraft HEPS. First of all ECMS cannot sustain the battery SoC on-line, and secondly they have not considered the aircraft safely landing since not keeping the battery SoC within a range.

The rule-based optimization is applied to reform the ECMS, since the control performance of the rule-based ECMS is not heavily dependent on the selection of equivalence factor [16,17]. Compared with the deterministic rule-based control, the Fuzzy Logic Control (FLC) is tolerant to imprecise measurements and variations, thereby being robust against system uncertainties [18]. The most important is that for the deterministic rule-based control, the battery SoC is typically sustained around a single value, using the classic set theory and deterministic rules. 
On the other hand, the inherent vagueness of FLC determines that the fuzzy control is appropriate for maintaining the SoC within a range, instead of a specific value.

As early as the 1990s, Lee and Sul applied the fuzzy logic decision-making to generate the motor torque command of the hybrid powered city bus [19]. The designed FLC achieved the $20 \%$ reduction of nitrogen oxides emission in the dynamometer test. Schouten, et al., demonstrated that better fuel economy and drivetrain efficiency could be achieved with the Takagi-Sugeno (T-S) fuzzy system for the ICE-dominated hybrid automobile [20]. It can be concluded from preceding studies that a FLC can maintain the SoC within a specified range and, furthermore, the T-S fuzzy system can decrease the computational complexity of the FLC [21,22]. The type-2 fuzzy logic system have emerged as a generalization of the type-1 fuzzy system. Researchers have started the application of type-2 fuzzy systems to the aerospace [23]. Castillo et al. compared the control performance between type-2 and type-1 fuzzy systems [24,25]. Those studies demonstrated that the generalized type-2 fuzzy based controller has better uncertainty and perturbation handling, but the computational complexity of type-2 fuzzy systems is still too intensive at the present time. Considering the practical applications, the type-1 fuzzy system is applied in this paper.

In order to solve the two deficiencies of ECMS, the paper proposes to integrate the fuzzy control into the conventional ECMS. The authors did not find in the open literature articles applying fuzzy based ECMS specifically to the aircraft HEPS. The paper is structured as follows. The high-level system architecture of the parallel hybrid powertrain is illustrated in Section 2. Section 3 presents the formulation of the energy management problem of the HEPS. The global optimization problem is converted to an instantaneous one using Hamiltonian function and its co-state variable. The equivalence factor is defined and ECMS method is established. In Section 4, the fuzzy logic control (fuzzy sets and fuzzy rules) is designed to regulate the battery SoC, in which the aircraft safety is considered. Section 5 combines the ECMS and fuzzy control to optimize the power split between the combustion and electric powertrains, while keeping the battery SoC in the desirable range. Section 6 reports the outcomes of two test cases in which the proposed fuzzy based ECMS is validated by comparing its performance with the conventional ECMS, Dynamic Programming (DP) and A-ECMS algorithms. Also, the relationship between the equivalence factor and co-state variable is verified via the hypothetical scenario.

\section{System Architecture}

There are various hybrid powertrain configurations currently in use. The most commonly used configurations are series, series-parallel and parallel architectures. Of these, the series architecture enables the engine to operate at its ideal operating condition. However, its system efficiency is relatively low since large energy losses exist in the energy conversion [26]. Also, the series configuration cannot achieve a combination of combustion and electrical power. The series-parallel is the most functional structure of the three, but its complicated clutch/gear mechanism raises the issue of reliability on real aircraft applications. Compared with the series-parallel configuration, the parallel configuration is lighter and less complex, whilst keeping the flexibility of hybridization. According to Hiserote's work [4], the parallel configuration is best suited for the long-endurance UAV.

A parallel HEPS was designed to act as the propulsion system for the aircraft, and its high-level component diagram is shown in Fig. 1. The engine is started using the integrated motor and a dedicated start battery. Once the engine has been started, its throttle is regulated by the Engine Control Unit (ECU), the integrated generator 
can power the payload via a DC/DC converter. The output shaft of the engine is connected through a reduction gear and sprag clutch to drive the motor/generator input shaft. The motor is powered by a second battery, and it can function as the generator to recharge that battery.

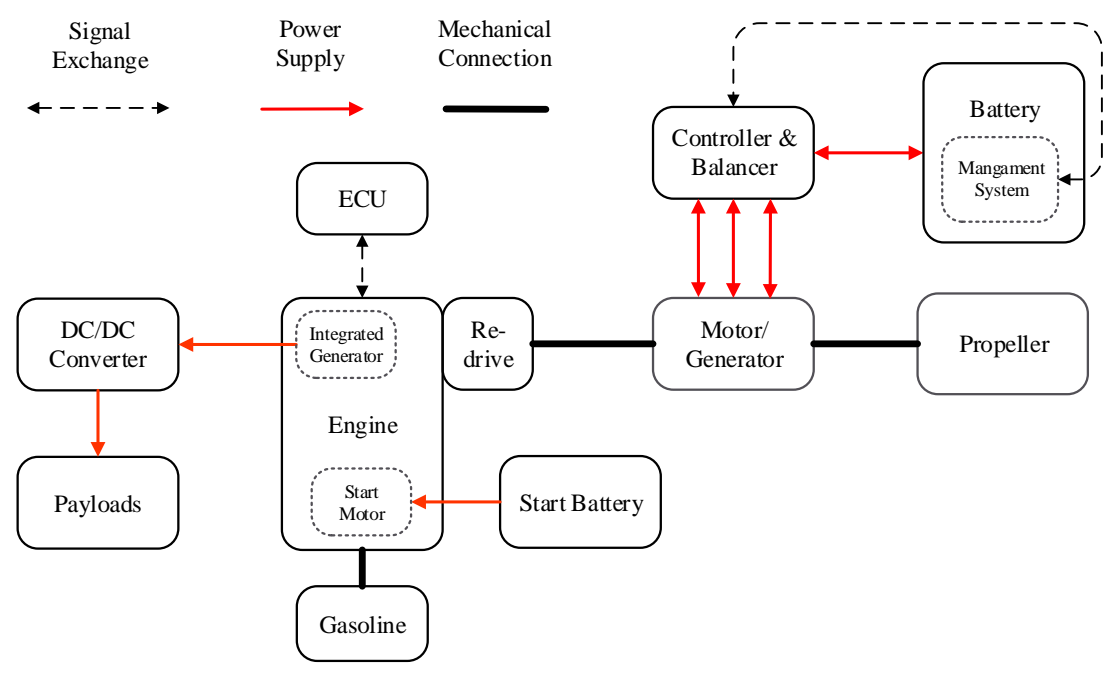

Fig. 1. Parallel hybrid propulsion system

This setup enables the system to combine the power from engine and motor/generator, when the power demand exceeds that which can be provided by only one of the propulsive units. Otherwise, when the power demand is lower than the engine maximum power, the motor/generator can draw power from the engine to charge the battery. Moreover, this architecture also permits the motor alone to drive the propeller, if electric-only flight mode is required. The hybrid architecture has the property that it is fault-tolerant. In the case of a failure of one of the propulsion units, it can de-couple the combustion drivetrain or shut down the electric power supply, so that one of the working units will continue driving the propeller. With energy management, the distribution of engine and motor/generator power can be optimized to improve fuel economy.

\section{Equivalent Consumption Management Strategy}

Initially, the formulation of the original problem, including constraints and the objective, will be presented. The internal battery power is introduced here to simplify the system dynamic constraint. Then, the global objective is converted to an instantaneous cost using the Hamiltonian function. The equivalent consumption method is developed by defining and introducing the equivalence factor.

\subsection{Problem Formulation}

\subsubsection{Constraints}

The role of HEPS energy management is to split the power demand appropriately between the combustion and electrical energy sources. Thus, the control variable of the powertrain is typically formulated as the engine output power, i.e. $u=P_{I C E}$. When selecting the battery for electrical energy storage, the state variable of the hybrid propulsion system is the battery's SoC, and is denoted by $x$. 
The formulated energy management problem is subject to the limitations of mission requirements, i.e. power requirements. The power demand $P_{r e q}$ usually appears as the sum of the engine power $P_{I C E}$ and motor/generator power $P_{M G}$ :

$$
P_{r e q}=P_{I C E}+P_{M G}
$$

where $P_{M G}$ is related to the battery power $P_{b a t t}$ by:

$$
P_{b a t t}=\left\{\begin{array}{ll}
\eta_{M G}^{-1} \cdot P_{M G}, & P_{M G}>0 \\
\eta_{M G} \cdot P_{M G}, & P_{M G}<0
\end{array} .\right.
$$

According to the definition of SoC, the system state transition equation can be written as:

$$
\dot{x}=-\frac{I}{Q_{\max }}
$$

where $I$ is the current flowing supplied by the battery and $Q_{\max }$ is the battery maximum capacity.

In hybrid vehicle analysis, the battery model is generally simplified to a pure resistance source [27]. The battery power can be expressed using current $I$, internal resistance $R_{\text {int }}$ and open circuit voltage $V_{b}$ :

$$
P_{\text {batt }}=I * V_{b}(x)-I^{2} R_{\text {int }}(x)
$$

The open circuit voltage $V_{b}$ depends on SoC and their relationship is given by Fig. 2. To extend the lifetime of the battery, the SoC is typically limited between two turning points, i.e. $20 \%$ and $80 \%$, respectively. The power related to the open circuit voltage is defined as the internal battery power $P_{b}$, i.e. $P_{b}=I * V_{b}(x)$.

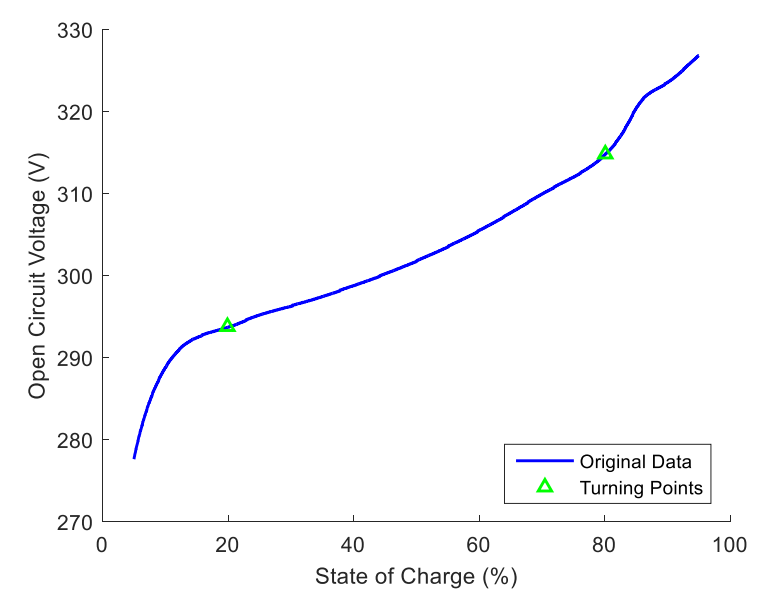

Fig. 2. Dependency of open circuit voltage on SoC

Generally, the internal resistance $R_{\text {int }}$ also varies with the change of SoC, but their relationship is normally unknown. In this paper, the power loss due to the internal resistance is denoted by $P_{b, l o s s}=I^{2} R_{\text {int }}(x)$. This power loss can be calculated using the experimental data and expressed by the internal battery power and battery SoC (see Fig. 3). As shown in Fig. 3, the power loss is correlated mainly with internal battery power, rather than SoC. The power loss can be fitted with a quadratic function: $P_{b, l o s s}=a_{l} P_{b}^{2}$ and the fitted coefficient is $a_{l}=3.24 *$ $10^{-6}$. 


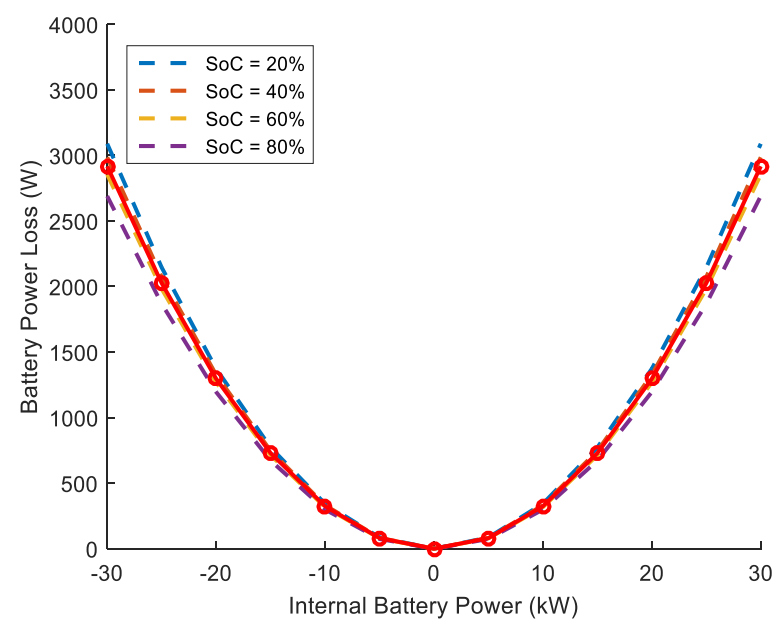

Fig. 3. Approximation of the battery power losses

If the internal battery power $P_{b}$, instead of battery power $P_{b a t t}$, is introduced in the system state equality, Eq. (3) can be addressed by:

$$
\dot{x}=-\frac{P_{b}}{V_{b}(x) \cdot Q_{\max }},
$$

in which the system state transition is affine in the battery internal power $P_{b}$.

In addition to system dynamics, the optimization must consider the physical constraints of each component, which could be expressed by the bounds of variables and given as Eqs. (6)-(8). Note that the engine rotational speed is $\omega_{I C E}$ and it is related to the motor/generator speed $\omega_{M G}$ via: $\omega_{I C E}=G * \omega_{\text {req }}$, where $G$ denotes the reduction gear ratio.

$$
\begin{gathered}
P_{I C E, \min }\left(\omega_{I C E}\right)<P_{I C E}<P_{I C E, \max }\left(\omega_{I C E}\right), \\
P_{M G, \min }\left(\omega_{M G}\right)<P_{M G}<P_{M G, \max }\left(\omega_{M G}\right), \\
S o C_{\min }<S o C<S o C_{\max } .
\end{gathered}
$$

\subsubsection{Objective}

Traditionally, the energy management of HEPS is formulated into a global optimization problem, of which objective is to minimize the fuel consumption for the complete mission:

$$
J=\int_{t_{0}}^{t_{f}} \dot{m}_{f} d t .
$$

where $\dot{m}_{f}$ denotes the fuel consumption rate of the engine. The time $t \in\left[t_{0}, t_{f}\right]$, where $t_{0}$ and $t_{f}$ are initial time and final time, respectively.

However, this objective requires unknown priori knowledge (like the complete flight mission). To avoid this drawback, ECMS approach proposes to replace the non-causal criterion by an instantaneous one [130], reducing the problem to a minimization of equivalent fuel consumption at any instant of time. 
If considering the energy management as the optimal control problem, the optimal control also minimizes the Hamiltonian function:

$$
H=\dot{m}_{f}+\lambda \cdot f(x, u)
$$

where $f(x, u)$ is the system state transition equation, i.e. $\dot{x}=f(x, u)$. The co-state variable $\lambda$ has to satisfy a dynamic equation defined in the maximum principle [28].

By substituting Eq. (5) for the system state transition equation $f(x, u)$ in Eq. (10), the Hamiltonian function can be transformed to:

$$
H=\dot{m}_{f}+\left(-\frac{\lambda}{V_{b}(x) \cdot Q_{\max }}\right) \cdot P_{b}
$$

If the term $-\frac{\lambda}{V_{b}(x) \cdot Q_{\max }}$ is replaced by the equivalence factor $s$, the Hamiltonian function can be considered as a new criterion [29]:

$$
J=\dot{m}_{\text {equiv }}=\dot{m}_{f}+s \cdot P_{b},
$$

where $\dot{m}_{\text {equiv }}$ is the equivalent consumption and $\dot{m}_{f}$ denotes the actual fuel consumption rate of the engine. The second term on the right of the equation $\left(s \cdot P_{b}\right)$ represents virtual fuel consumption rate of the electrical energy. The ECMS was initially established based on a charge-sustaining strategy, in which the battery is considered as an energy buffer with no net usage of electricity [29].

From above deducing, it is obvious that the co-state of Hamiltonian function, to some extent, represents the equivalence between the fuel and electric energy usage. If the equivalence factor has to be evaluated from the costate variable, ECMS will lose the advantage when compared with the maximum principle. The following text will present how to estimate the equivalence factor based on its physical meaning; while, the relationship between co-state $\lambda$ and equivalence factor $s$ will be demonstrated in Section 6.4.

\subsection{Equivalence Factor}

The equivalence factor represents the core of ECMS. This parameter influences the system behaviour as follows: if it is too large, the use of electrical energy tends to be penalized and the fuel consumption increases; if, on the contrary, it is too small, the use of electrical energy is overly favoured and the battery SoC decreases [29]. Since the equivalence factor represents the conversion ratio between two energies, it can be estimated using Specific Fuel Consumption (SFC). Given that the efficiencies of the electric path must be included, the equivalence factor can be expressed as:

$$
s= \begin{cases}S F C \cdot \eta_{M G}^{-1} \cdot \eta_{\text {batt.c }}^{-1}, & P_{b}>0 \\ S F C \cdot \eta_{M G} \cdot \eta_{\text {batt }, d}, & P_{b}<0\end{cases}
$$

where $\eta_{M G}$ is the efficiency of motor/generator. $\eta_{b a t t, c}$ and $\eta_{b a t t, d}$ are battery charging/discharging efficiencies, respectively. The efficiency of the converter (motor controller) is not considered. Note that the equivalence factor defined in this paper is not dimensionless, since the battery power $P_{b}$ is introduced in Eq. (12) instead of a virtual electric fuel cost $\dot{m}_{e}$. Both the SFC and motor/generator efficiency map are shown in Fig. 4. 


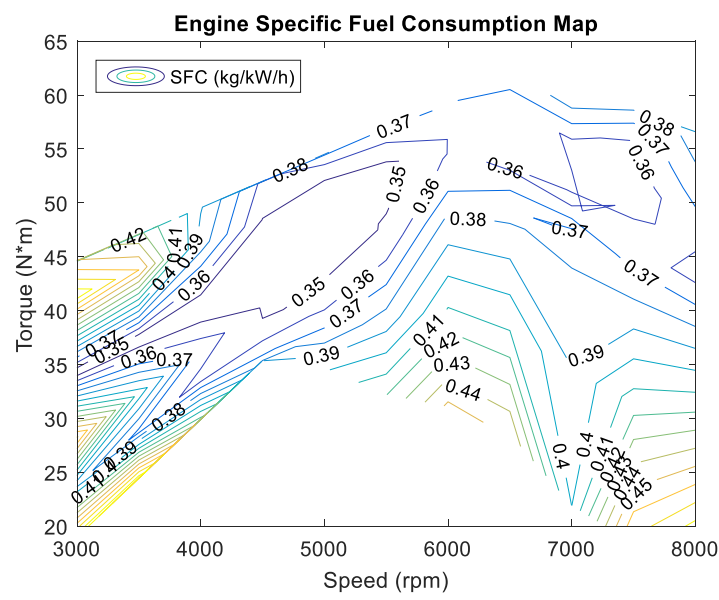

(a)

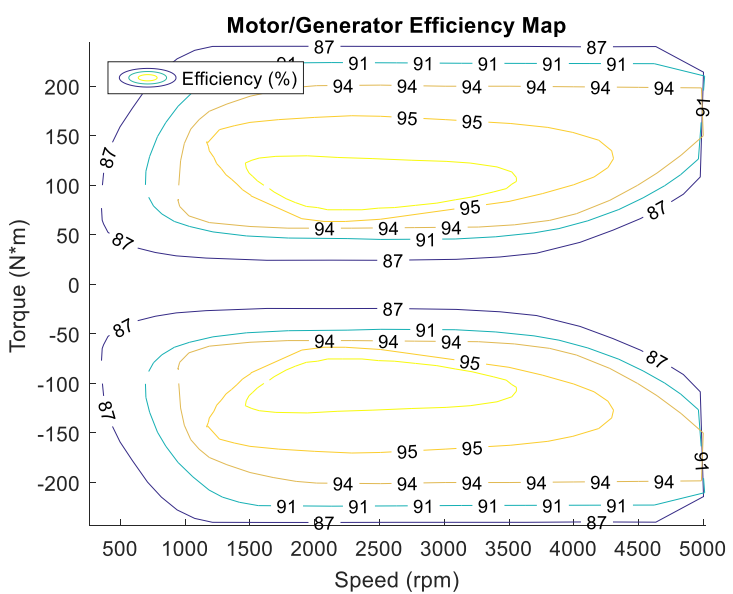

(b)

Fig. 4. Maps representing (a) specific fuel consumption, and (b) motor/generator efficiency

\section{Fuzzy Logic Control}

As mentioned above, the conventional ECMS cannot maintain the desired constraints on battery SoC. Therefore, a fuzzy logic controller has been designed to regulate the optimal control of ECMS according to the instantaneous value of the SoC. In this study, the fuzzy logic controller has four input variables: the power requirement $P_{r e q}$, the maximum engine power $P_{I C E, \max }$, the optimal engine power $P_{I C E, \text { opt }}$ and the SoC. While the output of the fuzzy controller is the motor/generator power $P_{M G}$.

A fuzzy logic controller generally consists of the rule-base, fuzzification interface, inference mechanism, and defuzzification interface [21]. The rule-base collects a series of rules that describe available expert knowledge and experience on how to control the system. On the fuzzification interface, the numeric inputs of the controller are converted into a fuzzy set. Then, using the fuzzy set and established rule-base, the inference mechanism mimics the expert's knowledge and determines the linguistic control decision. Lastly, defuzzification is performed to convert the linguistic control into actual control values (outputs of the controller).

\subsection{Fuzzy Sets}

The fuzzy set is introduced to represent fuzzy control inputs and outputs, to quantify the meaning of linguistic variables, linguistic values, and linguistic rules [21]. In addition, fuzzy sets are used to heuristically quantify the information in the rule-base and the operation of the inference mechanism. In this study, a functional fuzzy system (T-S) is introduced as the basis of the controller. In the same manner as a traditional fuzzy system, the T-S fuzzy system describes linguistic inputs by fuzzy sets. However, its consequent of rules is determined by the function with input variables as arguments.

A fuzzy set for each input is designed by defining the corresponding Membership Functions (MFs). The rulebase in this study needs the instantaneous value of four inputs, i.e. the power requirement, the maximum engine power, the optimal engine power, and the SoC. Using the power requirement, the maximum power and optimal power of the engine can be substituted by their difference. Thus, hereafter, the number of inputs is reduced to 
three and fewer MFs are required to cover the range of engine power. In other words, the initial four input variables are replaced by $P_{\text {req }}-P_{I C E, \text { max }}, P_{\text {req }}-P_{I C E, \text { opt }}$ and $S o C$ (see Fig. 5). In terms of $P_{\text {req }}-P_{I C E, \text { max }}$ and $P_{\text {req }}-$ $P_{I C E, \text { opt }}$, only two MFs are needed for each input since the range is divided into two regions ('neg' and 'pos'). These two inputs vary in the range of $(-30,30) \mathrm{kW}$ and $(-40,40) \mathrm{kW}$, respectively.
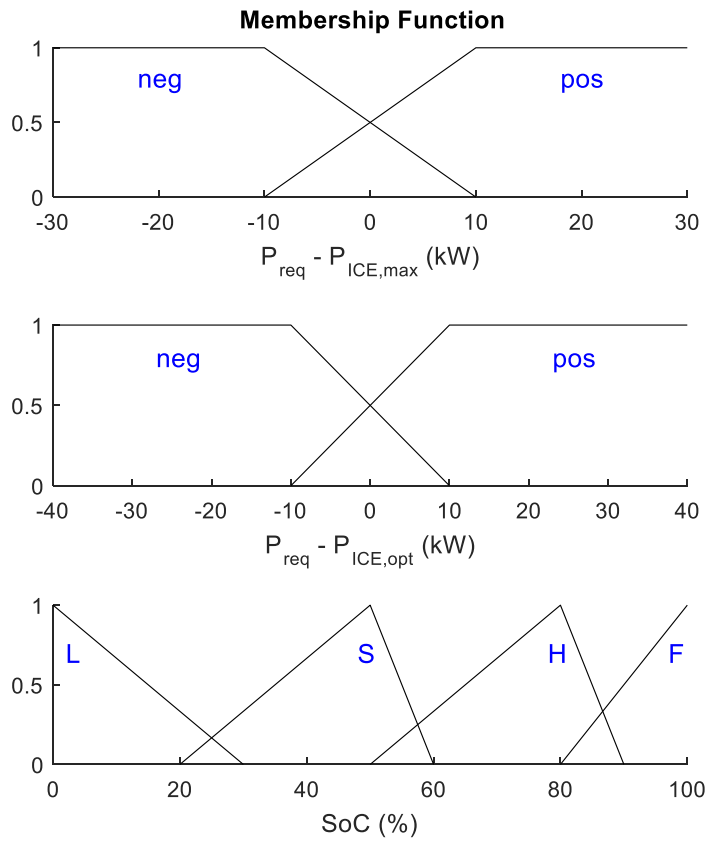

Fig. 5. Membership Functions

Concerning the $\mathrm{SoC}$, four linguistic terms are defined to represent values of $\mathrm{SoC}$, i.e. ' $\mathrm{L}$ ', 'S', 'H', and ' $\mathrm{F}$ '. The ' $\mathrm{L}$ ' is where the $\mathrm{SoC}$ is low and battery charging is requested if the extra engine power is available. The ' $\mathrm{S}$ ', denoting 'sustained', is the most important criterion, when the degree of this membership reaches 1 , the corresponding numeric value of $\mathrm{SoC}$ is the ideal sustainable value. The ' $\mathrm{H}$ ' implies that the $\mathrm{SoC}$ is high and charging is not compulsory, unless the power demand is lower than the optimal engine power. The full SoC is indicated by the ' $F$ ', where charging is not permitted.

It can be concluded from Fig. 2 that the battery voltage will decrease sharply when the SoC is under $20 \%$. Hence, the zero degree of ' $\mathrm{L}$ ' is set at slight above $20 \%$ SoC. On the other hand, $50 \%$ is selected as the ideal sustainable SoC, because the $50 \%$ electrical energy in the battery could guarantee a safe landing if the engine fails. The full advantage of combining engine and motor power can be obtained by classifying the range for fuzzy set ' $\mathrm{H}$ ' as $(50 \%, 90 \%)$.

\subsection{If-Then Rules}

The rule-base of fuzzy controller consists of a series of linguistic rules and called as If-Then rules. As above mentioned, the T-S fuzzy system is applied in this study. Therefore, the premises of the If-Then rules are represented by fuzzy sets with the associated MFs, while the consequents are approximated using linear functions:

1) If $P_{\text {req }}-P_{I C E, \max }$ is pos and $S o C$ is $\mathrm{H}$ or $S o C$ is $\mathrm{F}$, then $P_{M G}=P_{\text {req }}-P_{I C E, \text { opt }}$;

2) If $P_{\text {req }}-P_{I C E, \max }$ is pos and $S o C$ is $\mathrm{S}$, then $P_{M G}=P_{\text {req }}-P_{I C E, \max }$;

3) If $P_{\text {req }}-P_{I C E, \text { opt }}$ is neg and $S o C$ is F, then $P_{M G}=0$; 
4) If $P_{\text {req }}-P_{I C E, o p t}$ is neg and $S o C$ is not $\mathrm{F}$, then $P_{M G}=P_{\text {req }}-P_{I C E, \text { opt }}$;

5) If $P_{\text {req }}-P_{I C E, \max }$ is neg and $P_{\text {req }}-P_{I C E, \text { opt }}$ is pos and $S o C$ is $\mathrm{H}$ or $S o C$ is $\mathrm{F}$, then $P_{M G}=P_{\text {req }}-$ $P_{I C E, \text { opt }}$

6) If $P_{\text {req }}-P_{I C E, \text { max }}$ is neg and $P_{\text {req }}-P_{I C E, \text { opt }}$ is pos and $S o C$ is $\mathrm{L}$ or $S o C$ is $\mathrm{S}$, then $P_{M G}=P_{\text {req }}-$ $P_{I C E, \max }$

7) If $S o C$ is L, then $P_{M G}=f(S o C)$.

As shown in Rules 1) and 2), if the power demand is above the ICE maximum power, the positive motor/generator power decreases when the SoC drops from ' $\mathrm{H}$ ' level to the ' $\mathrm{S}$ ' level. On the other hand, when the power demand is lower than the ICE optimal power, battery charging will be activated and continue until the SoC is near its maximum capacity (see Rules 3) and 4)). The third scenario is that the power demand is between the ICE optimal and maximum power, see Rules 5) and 6). If the SoC is high enough ('H' or 'F'), the controller demands positive power from the motor/generator, to enable the ICE to operate at its optimal condition. Otherwise, the motor/generator acts as a generator to restore the electrical energy in the battery, using the extra available power of the engine. Rule 7) illustrates that charging power increases at lower values of SoC.

\section{3 (De-)fuzzification and Inference}

On the basis of designed fuzzy sets and If-Then rules, the fuzzification, inference, and defuzzification can be employed in sequence to deduce the final control output. In the fuzzification process, actual inputs are transformed into fuzzy sets. The fuzzy sets $\hat{A}_{i}$ are produced with a membership function $\mu_{\widehat{A}_{i}}(x)$ defined in Fig. 5.

The inference mechanism has two basic tasks: the first one is called matching, determines the extent to which each rule is relevant to the current system state; secondly, the inference step draws conclusions using instantaneous inputs and If-Then rules. The membership value $\mu_{i}$ for the $i^{\text {th }}$ rule's premise can be formed as:

$$
\mu_{i}=\mu_{\hat{A}_{1}^{j}}\left(u_{1}\right) * \mu_{\hat{A}_{2}^{k}}\left(u_{2}\right) * \mu_{\hat{A}_{3}^{l}}\left(u_{3}\right),
$$

where $*$ denotes fuzzy intersection. The parameters $j, k, l$ represent all possible $j, k, l$ MFs for three input variables.

In the defuzzification process, the linear functions $\beta_{i}$ are given by the consequents of If-Then rules in Section 4.2. Therefore, the defuzzification is expressed as:

$$
y=\frac{\sum_{i=1}^{R} \beta_{i} \mu_{i}}{\sum_{i=1}^{R} \mu_{i}},
$$

where $y$ is the numeric output of the fuzzy controller. It is assumed that the functional fuzzy system is well defined so that $\sum_{i=1}^{R} \mu_{i} \neq 0$ for all possible inputs.

\section{Combined Supervisory Control}

With the combination of ECMS and FLC, our supervisory control is named Fuzzy based ECMS (F-ECMS). F-ECMS can achieve the optimal hybrid of fuel and electrical energy, and also keep the battery SoC in the desirable range. Fig. 6 gives an overview of the structure of the combined supervisory controller and HEPS. The details of the HEPS are ignored in the figure, as are several engine-related devices. The variable exchanges 
between the supervisory controller and HEPS are highlighted in this figure. The control signals from the supervisory controller to components are represented by dashed lines, while dotted lines denote variables that are fed back to the supervisory controller from components.

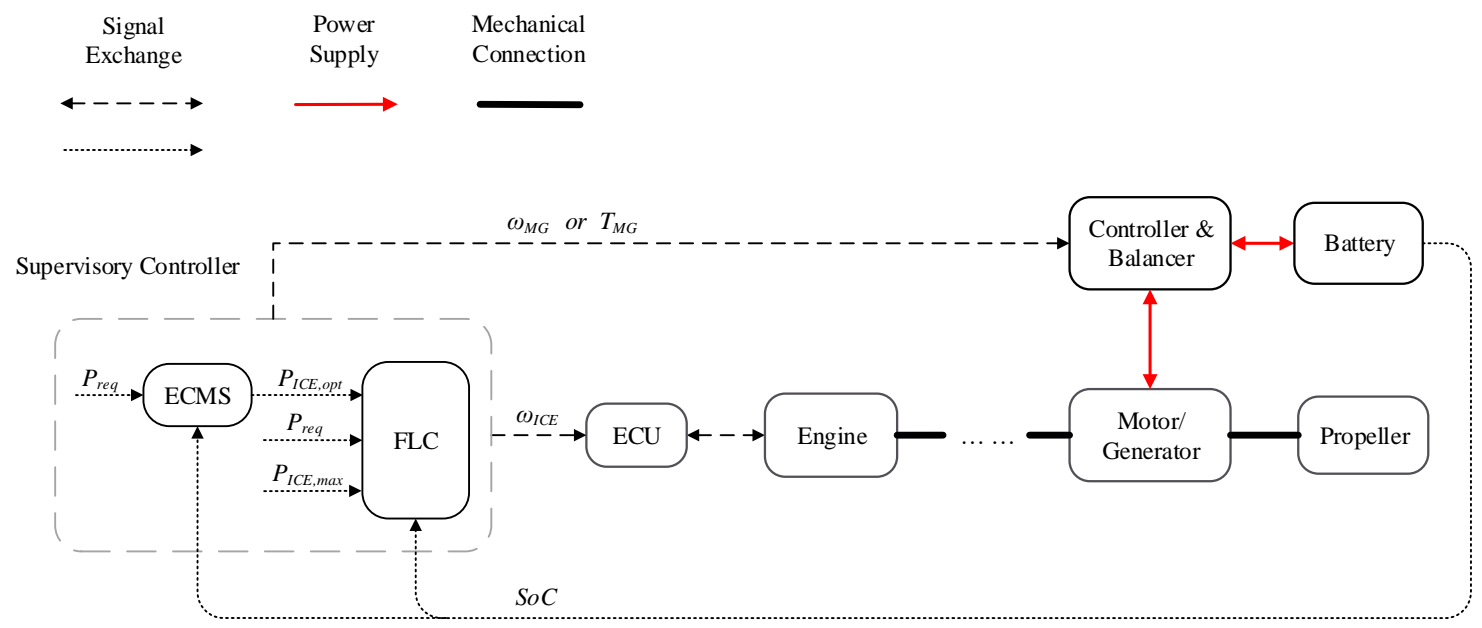

Fig. 6. Combined supervisory controller

As shown in Fig. 6, the ECMS requests the instantaneous value of power requirement $P_{r e q}$ and SoC to optimize the engine power. The optimal engine power from the ECMS is sent to the FLC, in addition to the maximum engine power, $P_{I C E, \max }$. The FLC still needs the power demand, $P_{r e q}$ and SoC to give the outputs of the supervisory controller. The outputs are motor/generator torque/speed command, $T_{M G} / \omega_{M G}$, and the engine speed command, $\omega_{I C E}$. Note that in this architecture, the power requirement, $P_{r e q}$, is the sum of the power requested to drive the propeller and power demanded by the auxiliary devices of the aircraft (such as payloads).

\section{Results and Analysis}

The work presented in this paper is part of the AIRSTART project to support the operation of the longendurance UAV equipped with advanced propulsion systems. Cranfield University is working on a hybrid propulsion system: converting a Rotron UAV engine into a hybrid combustion-electric system. The aerial platform for testing the hybrid propulsion system is an experimental medium-altitude long-endurance UAV-the Aegis.

This section reports the validation of the F-ECMS using two hypothetical flight scenarios. The advantages of the F-ECMS are validated by comparison with conventional ECMS, DP and A-ECMS. This section also validates the established relationship between the equivalence factor of ECMS and the co-state variable of Hamiltonian function. Please note that all simulations are carried out on the basis of the HEPS model developed in the previous study [15].

The key parameters of our HEPS are listed in Table 1, with their values and the corresponding units. Some of the parameters are explicitly used in the problem formulation, so their symbols are also given in the table. The rotation speed following a power value via @ indicates that this is the power reached at the stated speed. More details of system information and parameters can be acquired in the previous study [15]. 
Table 1 Parameters of Hybrid-Electric Propulsion System

\begin{tabular}{c|c|c}
\hline Parameter & Symbol & Value \\
\hline Maximum engine speed (rpm) & - & 7500 \\
Maximum engine power (kW) & - & $30 @ 6500 \mathrm{rpm}$ \\
Maximum motor speed (rpm) & - & 5000 \\
Maximum motor power (kW) & - & $30 @ 5000 \mathrm{rpm}$ \\
Nominal voltage $(\mathrm{V})$ & - & 296 \\
Capacity (Ah) & $Q_{\max }$ & 10 \\
Internal resistance $(\Omega)$ & $R_{\text {int }}$ & 0.6708 \\
Gear Ratio & $G$ & 2.5 \\
\hline
\end{tabular}

\subsection{Flight Missions}

In this paper, two flight missions are applied to validate the designed optimization algorithm. The mission case 1 used in this study simulates a complete 12-minute flight mission that is priori known. It includes take-off, climbing, cruising, and landing phases, see Fig. 7. The aircraft taxis and begins to takes-off in the first 1 minute. It then climbs to cruising altitude in the time interval of the $1^{\text {st }}-4^{\text {th }}$ minute after which it transitions to the cruising phase which commences at the $4^{\text {th }}$ minute. The aeroplane cruises at a constant speed and level flight for about five minutes until, at about the $9^{\text {th }}$ minute, the landing phase commences and then, finally, the mission ends at about the $13^{\text {th }}$ minute. The power requirement of this flight mission is as shown in Fig. 7.

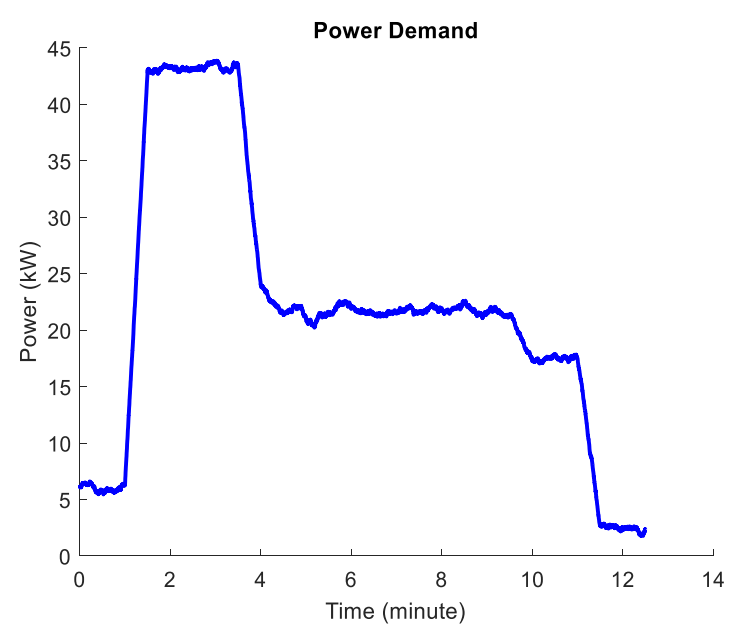

Fig. 7. Power requirement for flight mission 1

As shown in Fig. 7, the cruising phase is simplified to one segment with the relatively constant power requirement. To investigate the optimization performance during the cruising, this segment is re-designed and set as the second flight mission scenario. Different from the flight mission 1, the flight mission 2 is assumed to be previously unknown and excludes the take-off and landing phase. Its power requirement is as shown in Fig. 8. 


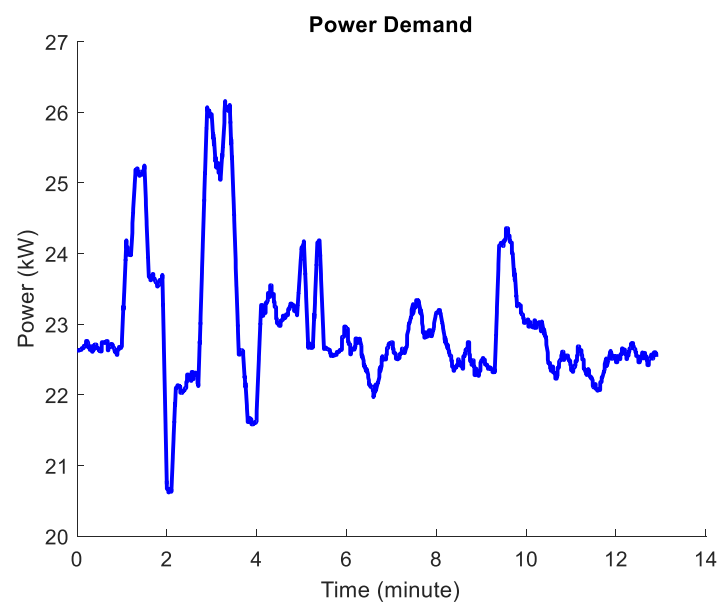

Fig. 8. Power requirement for flight mission 2

Since the first scenario is a priori-known flight mission, the DP can also be implemented; on the other hand, the second mission is assumed as impromptu and unpredictable, which can only be optimized by (A-) ECMS and our combined F-ECMS.

\subsection{Test Case 1}

In this test case, the results of conventional ECMS and DP are presented and are compared with the results of F-ECMS, under the first complete flight mission. Note that the mission is assumed to be a-prior known to the conventional ECMS and DP, but not to the F-ECMS. According to the If-Then rules of F-ECMS, the battery SoC should be sustained around 50\%. Thus, the final state of SoC is set as 50\% for the conventional ECMS and DP. The initial state is also $50 \%$ for three optimizations. The maximum and minimum value of battery SoC are $80 \%$ and $20 \%$, respectively.

Table 2 lists the information on the final $\mathrm{SoC}$ from three optimizations, in addition to the fuel/energy usage. It is obvious that the three approaches can ensure that the final value of battery $\mathrm{SoC}$ is around $50 \%$. Meanwhile, the error of DP is smaller than one of the conventional ECMS. Since the final SoC of three optimizations are not exactly the same, the direct comparison between fuel consumptions cannot reflect which one is better regarding the fuel saving. The SoC correction is introduced in this study to adjust the fuel consumption, since many researches [30] claim that fuel consumption in HEPS should be evaluated considering the balanced energy content of their electric storage devices.

The final SoC for both ECMS and F-ECMS is higher than for DP, which means DP implies greater consumption of electric energy. The extra electric energy stored in the battery with ECMS and F-ECMS is the cause of the additional fuel consumed. The real fuel consumption of three optimizations can be acquired by adjusting three final $\mathrm{SoC}$ values to the same one (50\%). This process is the above-mentioned SoC correction.

The corrected SoC and fuel usage are also displayed in Table 2. The corrected fuel consumption of DP is 1.67 $\mathrm{kg}$, while the corrected ones of ECMS and F-ECMS are basically the same. The error between the fuel usage of F-ECMS and the optimal one of DP is less than 3\%. In other words, F-ECMS can achieve sub-optimal results, though the fuzzy-based rules are applied to adjust optimal results from ECMS. 
Table 2 Comparison between ECMS, DP, and F-ECMS

\begin{tabular}{c|c|c|c|c}
\hline & \multirow{2}{*}{ Engine-Only } & \multicolumn{3}{|c}{ Hybrid } \\
\cline { 3 - 5 } & & ECMS & DP & F-ECMS \\
\hline Final SoC (\%) & - & 52.86 & 49.20 & 53.07 \\
Fuel Consumption (kg) & 1.93 & 1.72 & 1.67 & 1.72 \\
Corrected Final SoC (\%) & - & 50 & 50 & 50 \\
Corrected Fuel Consumption (kg) & - & 1.71 & 1.66 & 1.71 \\
Fuel Saving (\%) & 0 & 11.4 & 13.5 & 11.4 \\
Energy Consumption (MJ) & 89.63 & 80.15 & 77.27 & 79.49 \\
\hline
\end{tabular}

Furthermore, Table 2 compares the fuel burn between the hybrid propulsion system and combustion engineonly system, for the same flight mission. The optimal results from DP improve the fuel economy by $13.5 \%$, while our proposed F-ECMS can achieve up to $11.4 \%$ fuel reduction. The comparison of energy consumption between two different propulsion systems demonstrates that the hybrid propulsion system also consumes less total energy.

Fig. 9 gives the detailed results of three optimizations during the complete flight mission. The figure (a) provides the values of engine power and figure (b) presents the values of battery SoC. Under the charge-sustaining strategy, the conventional ECMS will maintain the SoC close to the sustaining value, regardless of the changes of power requirement during flight. Consequently, it cannot make full use of the electrical energy of the HEPS, since it only uses electrical energy as a buffer. Conversely, DP can benefit from hybridization, but its battery SoC is maintained around $40 \%$ during most of the flight time (between the $4^{\text {th }}$ and $10^{\text {th }}$ minute). Therefore, DP cannot guarantee enough electrical energy in the case of engine failure.

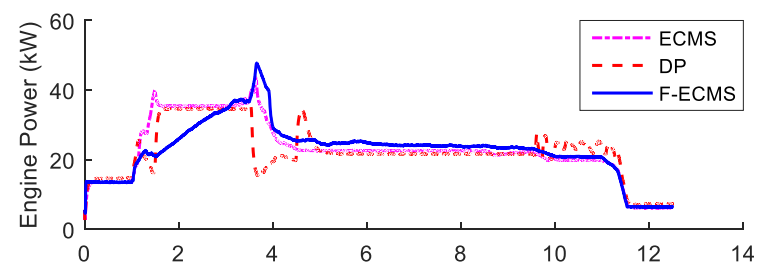

(a)

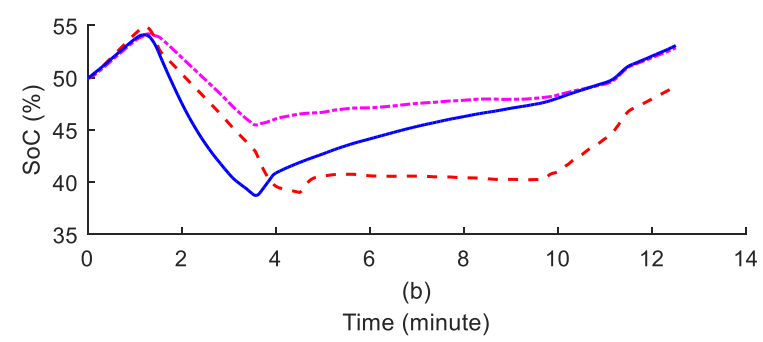

Fig. 9. Comparison between ECMS, DP, and F-ECMS, including engine power and battery SoC

However, the F-ECMS can solve this conflict by combining the conventional ECMS and fuzzy control. From the fuzzy logic rules of the F-ECMS, the SoC would be regulated toward one desired value (50\%) but also permitted to drop to a lower value if required. As shown in Fig. 9(a) and Fig. 9(b), though the SoC value is below $40 \%$ around the $4^{\text {th }}$ minute, electrical power is still available when there is a request for more power for a short 
duration (as for take-off and climb). In other words, the F-ECMS can not only keep the SoC within an acceptable range of the desired value, but also take advantage of the electrical energy source to power the aircraft.

Fig. 10 provides the details of fuel consumption and system efficiency. As shown in Fig. 10(a), the conventional ECMS consumes most fuel all of the time, while the fuel usage of F-ECMS becomes the same as one of ECMS at the end of the flight. During the first four minutes, DP has greater fuel consumption than FECMS, but after the $4^{\text {th }}$ minute DP uses less fuel than F-ECMS. This is the result of the charging process beginning from about the $4^{\text {th }}$ minute for the F-ECMS (see Fig. 9(b)). When the F-ECMS detects the SoC falling below $40 \%$ (and with the power demand declining), the F-ECMS commences supplying fuel energy to charge so that the SoC recovers. However, the DP does not charge the battery until the landing phase, since it recognizes that there will be no high power demand for the remaining part of the flight mission. The DP can gain the global optimal value because it has knowledge of the complete mission, but cannot be implemented on-line like F-ECMS.

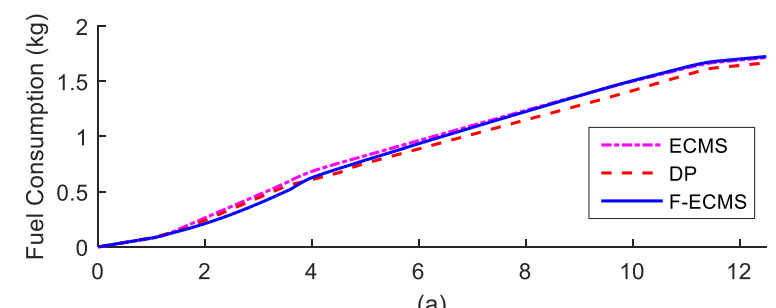

(a)

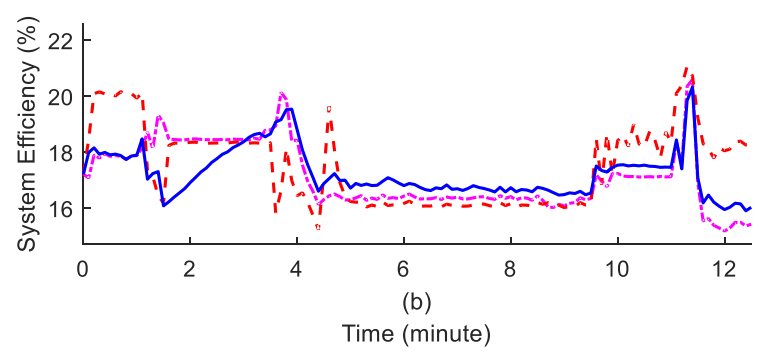

Fig. 10. Comparison between ECMS, DP, and F-ECMS, including fuel consumption and instantaneous system efficiency

Fig. 10(b) shows that the F-ECMS has the best efficiency during the cruise phase. It is common that the cruising phase, or phases having similar power requests, is normally the longest duration segment of a flight mission. Therefore, the gain on system efficiency from using F-ECMS will grow, with the increase in the duration of the flight mission.

In short, the F-ECMS succeeds in converting a global optimization into an equivalent instantaneous one, with little sacrifice of the optimality of the solution. In other words, the prior-knowledge of flight mission is not prerequisite and F-ECMS can achieve the sub-optimal control for on-line implementation. The most significant is that the F-ECMS can resolve the conflict between sustaining the SoC and electrical power usage, in comparison with the conventional ECMS and DP.

\subsection{Test Case 2}

The second flight mission and charge-sustaining strategy are implemented in this test case. Different from the test case 1, the second test case emphasizes the comparison of the computation cost between different instantaneous optimization algorithms and their capability of sustaining the SoC. Similarly, the initial and 
sustaining value of $\mathrm{SoC}$ is set as $50 \%$. In this test case, since the flight mission 2 is assumed to be previously unknown, the equivalence factor of the conventional ECMS is not optimized for the flight mission profile. In terms of A-ECMS, the equivalence factor is updated on-line using a PI controller. The formulation of this PI controller is illustrated in the following Section 6.4.

Table 3 lists the details of final values of SoC for ECMS, A-ECMS and F-ECMS, respectively. The SoC final values demonstrate that A-ECMS (49.94\%) and F-ECMS (49.96\%) can achieve charge-sustaining strategy without off-line optimization of equivalence factor. On the other hand, the conventional ECMS (43.83\%) performs worse regarding the SoC sustaining.

Table 3 Comparison between ECMS, A-ECMS, and F-ECMS

\begin{tabular}{c|c|c|c|c}
\hline & \multirow{2}{*}{ Engine-Only } & \multicolumn{3}{|c}{ Hybrid } \\
\cline { 3 - 5 } & & ECMS & A-ECMS & F-ECMS \\
\hline Final SoC (\%) & - & 43.83 & 49.94 & 49.96 \\
Fuel Consumption (kg) & 2.09 & 1.73 & 1.82 & 1.79 \\
Corrected Final SoC (\%) & - & 50 & 50 & 50 \\
Corrected Fuel Consumption (kg) & - & 1.75 & 1.82 & 1.79 \\
Fuel Saving (\%) & 0 & 17.2 & 12.9 & 14.4 \\
Average Optimization Time (sec) & - & 0.0032 & 0.0078 & 0.0033 \\
$\begin{array}{c}\text { Need for Off-line Optimization of } \\
\text { Equivalence Factor }\end{array}$ & - & Yes & No & No \\
\hline
\end{tabular}

In addition, Table 3 provides the fuel consumption and its corrected value of the three instantaneous optimizations. The corrected fuel usages demonstrate that the conventional ECMS consumes the least fuel (1.73 $\mathrm{kg}$ ) among three approaches, but fails to sustain the battery SoC. Meanwhile, compared with the engine-only system, the hybrid propulsion system can save about $14.4 \%$ fuel consumption using the F-ECM, approximately $3 \%$ lower than the conventional ECMS. However, F-ECMS saves more fuel than A-ECMS.

The average computation time of optimization is also given in Table 3. It is shown that the average optimization time of the A-ECMS is over two times higher than another two instantaneous methods. However, the combination of fuzzy control and ECMS does not increase much of the computational cost compared with the conventional ECMS.

Fig. 11 presents the results of engine power, battery SoC, and computation time of the three optimizations. As shown in Fig. 11(b), the conventional ECMS gives a decreasing value of SoC rather than sustaining it. Since the estimated equivalence factor is smaller than the real, optimal one, the electrical energy is overly favoured and the SoC decreases. This also indicates that the performance of the conventional ECMS is dependent on the equivalence factor. However, the equivalence factor cannot be optimized precisely without a-priori knowledge of the complete flight mission, so the conventional ECMS is not suitable for practical application. 


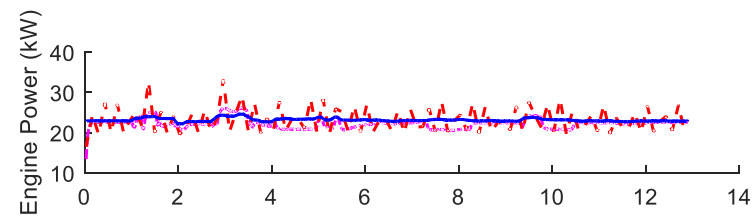

(a)

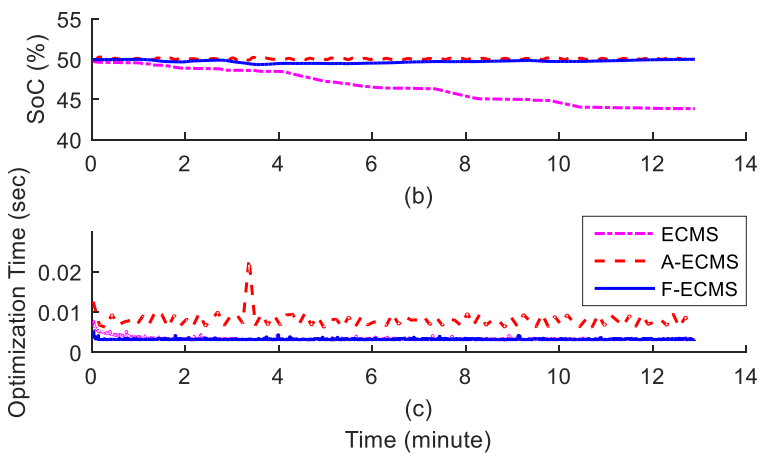

Fig. 11. Comparison between ECMS, A-ECMS, and F-ECMS, including engine power, battery SoC, and time of optimization

Fig. 11(b) also demonstrates that both A-ECMS and F-ECMS can achieve the charge-sustaining strategy without global information. The F-ECMS maintains the value of the SoC using fuzzy rules, while the A-ECMS can regulate the equivalence factor on-line to meet the requirements for maintaining the SoC. However, the FECMS is more practically useful than the A-ECMS, because the engine power of A-ECMS experiences more severe fluctuation than that of the F-ECMS (see Fig. 11(a)) and, in addition, the computational cost of A-ECMS is several times larger than that of the F-ECMS (see Fig. 11(c)). It is worth mentioning that the optimization time of F-ECMS has much the same value as the conventional ECMS.

In sum, the F-ECMS overcomes the deficiency of the conventional ECMS, by integrating fuzzy logic controller. Similar to A-ECMS, it can realize the on-line charge-sustaining with no need for off-line estimation of equivalence factor. Moreover, F-based ECMS outperforms A-ECMS, concerning the less computing time and less fuel consumption. Meanwhile, F-based ECMS results in a less fluctuation of the control value in comparison with A-ECMS. Another advantage is worth mentioning: it is well-known that A-ECMS algorithms are sensitive to the equivalence factor or penalty function for different flight missions [29]. This weakness is resolved in this study by combining fuzzy control with ECMS.

\subsection{Test Case 3}

As referred in Section 3.1.2, the equivalence factor of ECMS is related to the co-state variable of Hamiltonian function. The third test case aims to verify the relationship between the co-state variable and the equivalence factor. The Pontryagin's Maximum Principle (PMP) and A-ECMS are employed and compared under the first flight mission scenario. The co-state variable of PMP is transferred and compared with the equivalence factor of A-ECMS. The initial value of co-state variable and equivalence factor are both estimated by the cost-to-go of DP [31].

The A-ECMS updates the equivalence factor $s(t)$ by a PI controller to regulate the current SoC (i.e. $x(t)$ ) to the target value $x_{\text {sus }}: s(t)=s_{0}+K_{P}\left(x(t)-x_{\text {sus }}\right)+K_{I} \int\left(x(\tau)-x_{\text {sus }}\right) d \tau$. Here, $s_{0}$ is the initial value of equivalence factor, whereas $K_{P}$ and $K_{I}$ are gains of PI controller. Likewise, PMP takes the SoC into consideration 
using the state transition equation. The equivalence factor of PMP is defined and calculated using the co-state variable: $s(t)=-\frac{\lambda(t)}{V_{b}(t) \cdot Q_{\max }}$.

Fig. 12 compares the optimization time and equivalence factor between the PMP and A-ECMS. In terms of the computational cost, the A-ECMS has less optimization time than PMP (see Fig. 12 (a)). As shown in Fig. 12(b), the equivalence factor of PMP is clearly comparable to one of A-ECMS. The equivalence factor of PMP increases gradually and reaches a similar value as A-ECMS around the $4^{\text {th }}$ minute. This avoids the sharp up and down of equivalence factor, leading to a gently varying of optimal control.

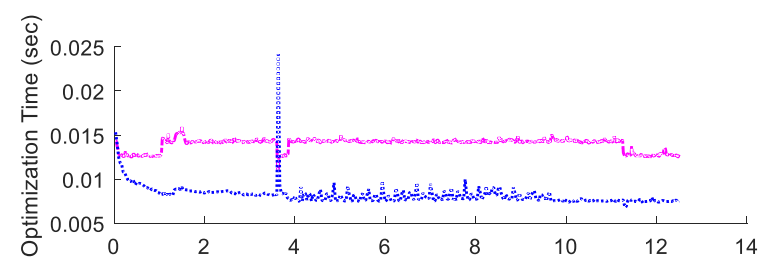

(a)

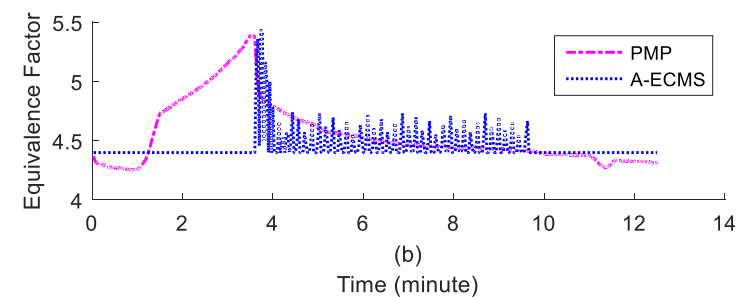

Fig. 12. Comparison between PMP and A-ECMS, including time of optimization and equivalence factor

In test case 3, the charge-depleting strategy is implemented. The initial value of SoC is set to $60 \%$. The maximum and minimum value of SoC are still $80 \%$ and $20 \%$, respectively. The battery SoC is allowed to be depleted to $30 \%$, then the energy management is demanded to sustain the SoC around this value. As shown in Fig. 13, both PMP and A-ECMS can achieve the charge-depleting strategy and their SoC trajectories are basically the same. On the other hand, the engine power (optimal control) of A-ECMS experiences more intense fluctuation with adaptively updated equivalence factor, in comparison with PMP.
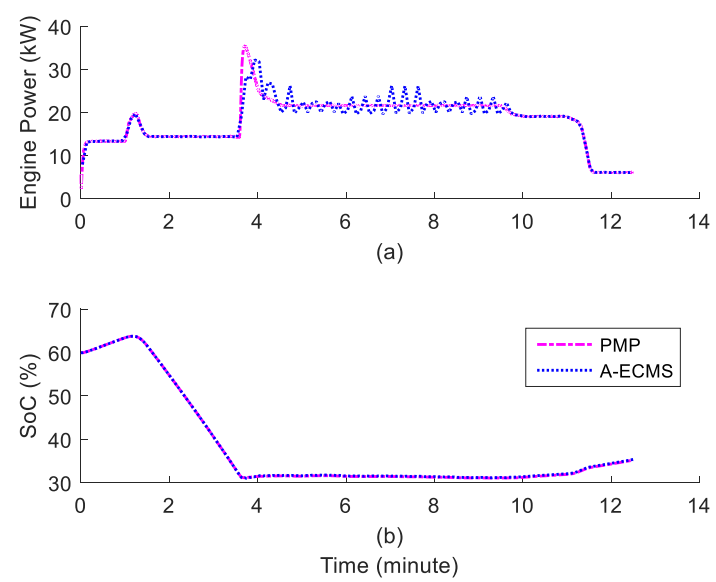

Fig. 13. Comparison between PMP and A-ECMS, including engine power and battery SoC 


\section{Conclusion}

This paper investigated the optimization of the energy management for UAV hybrid electric propulsion systems. The Hamiltonian function was introduced to transform the original global energy management problem into an instantaneous optimization problem. The ECMS approach and its equivalence factor were derived from the Hamiltonian function. The test case 3 validated that the relationship between the equivalence factor of ECMS and the co-state variable of Hamiltonian function can be represented by the equation provided in the paper. Since the conventional ECMS and its reformed approach (e.g. A-ECMS) are not ideal for aircraft, this paper presented an improvement of ECMS, using fuzzy control, for on-line applications.

The proposed approach, F-ECMS, was validated using two test cases: comparisons with the conventional ECMS, DP, and A-ECMS. The test case 1 demonstrated that the F-ECMS succeeded in converting the global optimization into an equivalent instantaneous one, without much compromise of the optimality. Moreover, the FECMS accomplished a balance between maintaining the SoC and electrical power use, while the conventional ECMS targeted to use the combustion power to sustain the SoC and DP preferred to consume the electrical energy as much as possible. For the first flight mission, the HEPS with F-ECMS could save the fuel consumption up to $11.4 \%$, in comparison with the combustion system. From the test case 2, the F-ECMS solve the issue of the conventional ECMS, and could accurately sustain the SoC within prescribed limits without a-priori knowledge of global mission. Additionally, the F-ECMS was superior to the A-ECMS in practical application, since it required less fuel consumption, less computation time, and less fluctuation of control values. Its fuel used for the second flight mission was $14.4 \%$ lower than the engine-only powered aircraft.

The paper has completed the design and simulation validation of an on-line energy optimization, F-ECMS, for the UAV hybrid propulsion system. The areas of future work are recommended as follows: 1) F-ECMS should be validated in the Hardware-in-the-Loop (HiL) system. The real-time capability is the main performance required to be validated. The optimization solvers for the embedded systems are needed. The on-line estimation of battery $\mathrm{SoC}$ is required to be integrated into the embedded systems. 2) Considering reducing the pollutant emissions of hybrid propulsion systems. The reduction of carbon monoxide and nitrogen oxides is worth studying to reduce the side effect of hybrid-powered aircraft on the environment. The emission maps of the engine are needed for this further study.

\section{Conflict of interest statement}

The authors declared that they have no conflicts of interest to this work.

\section{Acknowledgements}

This work was partly supported by the InnovateUK [grant number 102361], AIRSTART project which is a $£ 3.2$ million collaborative Research and Development project developing key technologies to support general aviation, which includes the development of advanced propulsion systems. The authors would like to thank the China Scholarship Council (CSC) for funding Ye Xie [grant number 201506830053] to pursue her Ph.D. degree at Cranfield University. 


\section{References}

[1] NASA Aeronautics Research Mission Directorate, Strategic Implementation Plan, 2017. https://www.nasa.gov/sites/default/files/atoms/files/sip-2017-03-23-17-high.pdf.

[2] M. Njeh, S. Cauet, P. Coirault, P. Martin, Hœ control strategy of motor torque ripple in hybrid electric vehicles: an experimental study, IET Control Theory Appl. 5 (2011) 131. doi:10.1049/iet-cta.2010.0036.

[3] F.G. Harmon, A.A. Frank, J. Chattot, Conceptual Design and Simulation of a Small Hybrid-Electric Unmanned Aerial Vehicle, J. Aircr. 43 (2006) 1490-1498. doi:10.2514/1.15816.

[4] R. Hiserote, F. Harmon, Analysis of Hybrid-Electric Propulsion System Designs for Small Unmanned Aircraft Systems, in: 8th Annu. Int. Energy Convers. Eng. Conf., 2010. doi:10.2514/6.2010-6687.

[5] J.Y. Hung, L.F. Gonzalez, On parallel hybrid-electric propulsion system for unmanned aerial vehicles, Prog. Aerosp. Sci. 51 (2012) 1-17. doi:10.1016/j.paerosci.2011.12.001.

[6] J. Schoemann, M. Hornung, Modeling of Hybrid Electric Propulsion Systems for Small Unmanned Aerial Vehicles, in: 12th AIAA Aviat. Technol. Integr. Oper. Conf. 14th AIAA/ISSMO Multidiscip. Anal. Optim. Conf., American Institute of Aeronautics and Astronautics, Reston, Virigina, 2012: p. 5610. doi:10.2514/6.2012-5610.

[7] P. Panagiotou, I. Tsavlidis, K. Yakinthos, Conceptual design of a hybrid solar MALE UAV, Aerosp. Sci. Technol. (2016). doi:10.1016/j.ast.2016.03.023.

[8] C. Friedrich, P.A. Robertson, Hybrid-Electric Propulsion for Aircraft, J. Aircr. 52 (2015) 176-189. doi:10.2514/1.C032660.

[9] C.E.D. Riboldi, An optimal approach to the preliminary design of small hybrid-electric aircraft, Aerosp. Sci. Technol. 81 (2018) 14-31. doi:10.1016/j.ast.2018.07.042.

[10] G. Paganelli, T.M. Guerra, S. Delprat, J.-J. Santin, M. Delhom, E. Combes, Simulation and assessment of power control strategies for a parallel hybrid car, Proc. Inst. Mech. Eng. Part D J. Automob. Eng. 214 (2000) 705-717. doi:10.1243/0954407001527583.

[11] C. Musardo, G. Rizzoni, Y. Guezennec, B. Staccia, A-ECMS: An Adaptive Algorithm for Hybrid Electric Vehicle Energy Management, Eur. J. Control. 11 (2005) 509-524. doi:10.3166/ejc.11.509-524.

[12] B. Geng, J.K. Mills, D. Sun, Energy management control of microturbine-powered plug-in hybrid electric vehicles using the telemetry equivalent consumption minimization strategy, IEEE Trans. Veh. Technol. 60 (2011) 4238-4248. doi:10.1109/TVT.2011.2172646.

[13] J. Han, D. Kum, Y. Park, Synthesis of Predictive Equivalent Consumption Minimization Strategy for Hybrid Electric Vehicles Based on Closed-Form Solution of Optimal Equivalence Factor, IEEE Trans. Veh. Technol. 66 (2017) 5604-5616. doi:10.1109/TVT.2017.2660764.

[14] S. Onori, L. Serrao, G. Rizzoni, Adaptive Equivalent Consumption Minimization Strategy for Hybrid Electric Vehicles, in: ASME 2010 Dyn. Syst. Control Conf. Vol. 1, ASME, 2010: pp. 499-505. doi:10.1115/DSCC2010-4211.

[15] Y. Xie, A. Savvaris, A. Tsourdos, J. Laycock, A. Farmer, Modelling and control of a hybrid electric propulsion system for unmanned aerial vehicles, in: 2018 IEEE Aerosp. Conf., IEEE, Bozeman, 2018: pp. 1-13. doi:10.1109/AERO.2018.8396436.

[16] T. Hofman, R.M. van Druten, M. Steinbuch, A.F.A. Serrarens, Rule-Based Equivalent Fuel Consumption Minimization Strategies for Hybrid Vehicles, IFAC Proc. Vol. 41 (2008) 5652-5657. doi:10.3182/20080706-5-KR-1001.00953.

[17] B. Skugor, J. Deur, M. Cipek, D. Pavkovi, Design of a power-split hybrid electric vehicle control system utilizing a rule-based controller and an equivalent consumption minimization strategy, Proc. Inst. Mech. Eng. Part D J. Automob. Eng. 228 (2014) 631-648. doi:10.1177/0954407013517220.

[18] Ho Jae Lee, Jin Bae Park, Guanrong Chen, Robust fuzzy control of nonlinear systems with parametric uncertainties, IEEE Trans. Fuzzy Syst. 9 (2001) 369-379. doi:10.1109/91.919258.

[19] Hyeoun-Dong Lee, Seung-Ki Sul, Fuzzy-logic-based torque control strategy for parallel-type hybrid electric vehicle, IEEE Trans. Ind. Electron. 45 (1998) 625-632. doi:10.1109/41.704891.

[20] N.J. Schouten, M.A. Salman, N.A. Kheir, Fuzzy logic control for parallel hybrid vehicles, IEEE Trans. 
Control Syst. Technol. 10 (2002) 460-468. doi:10.1109/87.998036.

[21] K.M. Passino, S. Yurkovich, Fuzzy Control, Addison-Wesley, 1998. doi:10.3233/978-1-60750-706-239.

[22] M.A. Sancheza, O. Castillo, J.R. Castroa, Information granule formation via the concept of uncertaintybased information with Interval Type-2 Fuzzy Sets representation and Takagi-Sugeno-Kang consequents optimized with Cuckoo search, Appl. Soft Comput. J. (2015). doi:10.1016/j.asoc.2014.05.036.

[23] O. Castillo, L. Cervantes, J. Soria, M. Sanchez, J.R. Castro, A generalized type-2 fuzzy granular approach with applications to aerospace, Inf. Sci. (Ny). (2016). doi:10.1016/j.ins.2016.03.001.

[24] M.A. Sanchez, O. Castillo, J.R. Castro, Generalized Type-2 Fuzzy Systems for controlling a mobile robot and a performance comparison with Interval Type-2 and Type-1 Fuzzy Systems, Expert Syst. Appl. (2015). doi:10.1016/j.eswa.2015.03.024.

[25] O. Castillo, L. Amador-Angulo, J.R. Castro, M. Garcia-Valdez, A comparative study of type-1 fuzzy logic systems, interval type-2 fuzzy logic systems and generalized type-2 fuzzy logic systems in control problems, Inf. Sci. (Ny). (2016). doi:10.1016/j.ins.2016.03.026.

[26] C.C. Chan, The State of the Art of Electric, Hybrid, and Fuel Cell Vehicles, Proc. IEEE. 95 (2007) $704-$ 718. doi:10.1109/JPROC.2007.892489.

[27] C. Zhang, Y. Zhang, X. Zhang, State-of-charge estimation of the lithium-ion battery system with timevarying parameter for hybrid electric vehicles, IET Control Theory Appl. 8 (2014) 160-167. doi:10.1049/iet-cta.2013.0082.

[28] L.D. Berkovitz, Optimal Control Theory, Springer Science+Business Media, LLC, 1974.

[29] G. Paganelli, General supervisory control policy for the energy optimization of charge-sustaining hybrid electric vehicles, JSAE Rev. 22 (2001) 511-518. doi:10.1016/S0389-4304(01)00138-2.

[30] C. Hou, M. Ouyang, L. Xu, H. Wang, Approximate Pontryagin's minimum principle applied to the energy management of plug-in hybrid electric vehicles, Appl. Energy. 115 (2014) 174-189. doi:10.1016/j.apenergy.2013.11.002.

[31] C. Zhang, A. Vahidi, Route preview in energy management of plug-in hybrid vehicles, IEEE Trans. Control Syst. Technol. 20 (2012) 546-553. doi:10.1109/TCST.2011.2115242. 
Fuzzy logic based equivalent consumption optimization of a hybrid electric propulsion system for unmanned aerial vehicles

Xie, Ye

Elsevier

Xie Y, Savvaris A, Tsourdos A. (2019) Fuzzy logic based equivalent consumption optimization of a hybrid electric propulsion system for unmanned aerial vehicles. Aerospace Science and Technology, Volume 85, February 2019, pp. 13-23

https://doi.org/10.1016/j.ast.2018.12.001

Downloaded from Cranfield Library Services E-Repository 